\begin{tabular}{|c|c|c|}
\hline $\begin{array}{l}\text { PUCRS } \\
\text { PUCR }\end{array}$ & $\begin{array}{l}\text { ESCOLA DE CIÊNCIAS } \\
\text { DA SAÚDE E DA VIDA }\end{array}$ & $\begin{array}{l}\text { Psico, Porto Alegre, v. 52, n. 3, p. 1-14, jul.-set. } 2021 \\
\text { e-ISSN: } 1980-8623 \text { | ISSN-L: 0103-5371 }\end{array}$ \\
\hline (क) http:// & doi.org/10.15448/1980-8623.2021.3.39894 & \\
\hline
\end{tabular}

SEÇÃO: ARTIGO

\title{
Redes sociais e apoio social em estudantes de enfermagem durante a pandemia covid-19: estudo transversal
}

\author{
Social networks and social support in nursing students during the covid-19 pandemic: \\ cross-sectional study
}

Redes sociales y apoyo social en estudiantes de enfermeria durante la pandemia covid-19: estudio transversal

Romario Daniel Jantara ${ }^{1}$ orcid.org/0000-0002-7417-499X romario.jantara@outlook.com

\section{Daiane Porto Gautério \\ Abreu $^{1}$ \\ orcid.org/0000-0002-1125-4693 \\ daianeportoabreu@gmail.com}

\section{Leni de Lima Santana ${ }^{2}$ \\ orcid.org/0000-0001-5480-7684 leni.santana@ifpr.edu.br}

Diéssica Roggia Piexak ${ }^{1}$ orcid.org/0000-0002-3374-7843 diessicap@yahoo.com.br

\section{Stella Minasi de Oliveira $^{1}$}

orcid.org/0000-0002-1363-717X isminasi@yahoo.com.br

Recebido em: 12 jan. 2021. Aprovado em: 1 jul. 2021 Publicado em: 27 out. 2021.

\section{(c) (i)}

Artigo está licenciado sob forma de uma licença Creative Commons Atribuicão 4.0 Internacional.
Resumo: O objetivo deste estudo é identificar os tipos de redes sociais acessadas e a percepção do apoio social recebido por graduandos de enfermagem no contexto da pandemia de covid-19. Trata-se de um estudo transversal, descritivo e correlacional, realizado com 147 estudantes de graduação em enfermagem de uma universidade federal. A coleta de dados ocorreu por meio de formulário eletrônico, contendo questionário de caracterização de elaboração própria, Escala de Solidão, Escala de Apoio Social e Escala de Depressão, Ansiedade e Estresse. As principais redes sociais identificadas foram as familiares, as de amizade e as institucionais. Prevaleceu nivel médio de apoio social para as dimensões apoio emocional/informacional (56.5\%), interação social (47,6\%) e alto nivel para as dimensões apoio material $(41,5 \%)$ e apoio afetivo $(51,7 \%)$. Embora o estudo tenha identificado as redes sociais e a percepção de apoio social, mais pesquisas são necessárias para esclarecer os impactos da pandemia na população estudada. Palavras-chave: apoio social, redes sociais, estudantes de enfermagem, coronavirus, ensino

Abstract: The aim of this study is to identify the types of social networks accessed and the perception of social support received by nursing students in the context of the covid-19 pandemic. This is a cross-sectional, descriptive and correlational study carried out with 147 undergraduate nursing students from a federal university. Data collection took place through an electronic form, containing a self-designed characterization questionnaire, Loneliness Scale, Social Support Scale and Depression, Anxiety and Stress Scale. The main social networks identified were family networks, friendship networks and institutional networks. Average level of social support prevailed for the dimensions emotional/informational support $(56.5 \%)$, social interaction (47.6\%) and high level for the dimension's material support $(41.5 \%)$ and affective support (51.7\%). Although the study identified social networks and the perception of social support, more research is needed to clarify the impacts of the pandemic in the studied population.

Keywords: social support, social networks, nursing students, coronavirus, teaching

Resumen: El objetivo de este estudio es identificar los tipos de redes sociales a las que se accede y la percepción del apoyo social que reciben los estudiantes de enfermería en el contexto de la pandemia de covid-19. Se trata de un estudio transversal, descriptivo y correlacional, realizado con 147 estudiantes de pregrado en enfermería de una universidad federal. La recolección de datos se realizó a través de un formulario electrónico, que contiene un cuestionario de caracterización de diseño propio, Escala de Soledad, Escala de Apoyo Social y Escala de Depresión, Ansiedad y Estrés. Las principales redes sociales identificadas fueron redes familiares, redes de amistad y redes institucionales. Predominó el nivel 
medio de apoyo social para las dimensiones apoyo emocional / informativo (56,5\%), interacción social $(47,6 \%)$ y nivel alto para las dimensiones apoyo material $(41,5 \%)$ y apoyo afectivo $(51,7 \%)$. Si bien el estudio identificó las redes sociales y la percepción de apoyo social, se necesita más investigación para esclarecer los impactos de la pandemia en la población estudiada.

Palabras clave: apoyo social, redes sociales, estudiantes de enfermeria, coronavirus, enseñanza

A espécie humana é fundamentalmente social, sendo que é inerente ao ser humano a necessidade de reconhecer, interagir e formar relações entre os pares. Ao longo do tempo, evidências substanciais acumuladas determinaram que as relações sociais têm grande relevância para o bem-estar físico e mental das pessoas (Cacioppo \& Cacioppo, 2014). A conexão social é essencial para o desenvolvimento do homem, sua saúde e sobrevivência, sendo que, possivelmente, não existam outros fatores que possam ter um impacto tão grande na duração e na qualidade de vida quanto os laços sociais formados. Estar conectado socialmente reduz significativamente o risco de mortalidade prematura, enquanto a falta de conexão é capaz de aumentar este risco (Holt-Lunstad et al., 2017).

Conceitualmente, rede social é definida como o grupo de pessoas com as quais o individuo mantém contato ou alguma forma de vínculo social (Sherbourne \& Stewart, 1991). Podem se caracterizar como redes familiares, de amizade, vizinhança e institucional (Guadalupe \& Vicente, 2020). Em contrapartida, o apoio social se refere aos recursos postos à disposição de um individuo por outras pessoas em situações de necessidade (Zanini et al., 2017). Dispõe-se que a rede social é considerada uma dimensão estrutural das relações sociais, sendo entendida como uma estrutura pela qual o apoio social é ou deveria ser fornecido, enquanto o apoio social é caracterizado como a dimensão funcional dos relacionamentos (Griep, 2003; Griep et al., 2005).

Podem ser identificadas distintas dimensões do apoio social, a saber: emocional; informacional; material; de interação social positiva; e afetivo. Apoio material se relaciona com a provisão de recursos práticos e ajuda material; enquanto o afetivo,a demonstrações físicas de amor e afeto; o emocional a expressões de afeto positivo, compreensão e sentimentos de confiança; a interação social positiva diz respeito à disponibilidade de pessoas para se divertirem ou relaxarem; e o apoio informacional a disponibilidade de pessoas para a obtenção de conselhos ou orientações (Sherbourne \& Stewart, 1991).

As redes sociais têm importantes implicações em momentos da vida de mudanças que exigem adaptações, como os vivenciados durante a formação acadêmica. No meio universitário, os estudantes encontram-se expostos a desafios culturais, sociais, e econômicos, dentre outros, que podem desencadear vulnerabilidades ao sofrimento psicológico. Assim, o apoio social ocupa uma função significativa no enfrentamento dos aspectos desafiadores da vida universitária (Jantara et al., 2020).

No contexto da pandemia de covid-19, já são observados efeitos psicológicos e sociais diretos e indiretos, que se caracterizam pela sua generalidade e por afetarem, principalmente, a saúde mental das pessoas (Holmes et al., 2020). A área da educação sofreu profundamente com seus impactos, uma vez que ocorreram fechamentos de instituições de ensino em mais de 194 países, afetando mais de $91 \%$ dos alunos matriculados em todo o mundo (United Nations Educational, Scientific and Cultural Organization [UNESCO], 2020). Delimita-se que o papel dos recursos sociais neste cenário emerge como uma das prioridades de pesquisa (Holmes et al., 2020).

Observa-se, assim, que, em virtude da suspensão das aulas presenciais e da imposição de medidas restritivas de contato social, o contexto pandêmico afetou diretamente as atividades acadêmicas dos estudantes de enfermagem, haja visto que parte significativa de suas aulas possuem caráter teórico-prático ou prático. Pressupõe-se, assim, que esse contexto pode ter afetado negativamente o acesso dos estudantes de enfermagem às suas redes sociais convencionais, modificando a estrutura das mesmas e, por conseguinte, diminuido a percepção do apoio social recebido.

Desse modo, considerando os impactos da pandemia, a abordagem da presente temática em estudos é urgente, principalmente no Brasil, 
devido ao fato de ser uma área de estudo ainda pouco explorada. Nesse sentido, delimitou-se como questões de pesquisa deste estudo: Quais os tipos de redes sociais que os estudantes universitários de enfermagem acessaram no contexto da pandemia de covid-19? Qual a percepção do apoio social recebido por estudantes universitários de enfermagem durante a pandemia de covid-19?

Assim, o objetivo deste estudo foi identificar os tipos de redes sociais acessadas e a percepção do apoio social recebido por graduandos de enfermagem no contexto da pandemia de covid-19.

\section{Método}

\section{Tipo de estudo e aspectos éticos}

Pesquisa quantitativa, descritiva, correlacional e de corte transversal. Obteve-se aprovação do Comitê de Ética em Pesquisa da Universidade Federal do Rio Grande (FURG), sob o parecer número 4.276.996 e Certificado de Apresentação para Apreciação Ética (CAAE) número 37158020.9.0000.5324. Todos os aspectos éticos das pesquisas que envolvem seres humanos foram respeitados, em consonância com a Resolução n 510/2016 (Resolução n 510, 2016).

\section{Participantes}

Os participantes deste estudo são os estudantes matriculados no curso de graduação em enfermagem da FURG. Foram critérios de inclusão: possuir 18 anos ou mais e estar regularmente matriculado no referido curso e o critério de exclusão foi: não possuir acesso à internet, pois a coleta foi realizada de forma remota. Acrescenta-se que no período de realização de estudo a universidade já possuía programa de inclusão social que disponibilizava internet móvel gratuita para os estudantes, o que possibilitava a participação de todos os estudantes.

Segundo o Núcleo de Tecnologia da Informação (NTI) da universidade na qual foi realizado o estudo o número total de estudantes regularmente matriculados no segundo semestre de 2020 era de 278. Foi realizado um cálculo de amostra para populações finitas em estudos transversais: $\mathrm{n}=(\mathrm{Z} 2 \times \mathrm{P} \times \mathrm{Q} \times \mathrm{N}) /(\mathrm{e} 2 \times(\mathrm{N}-1)+\mathrm{Z} 2 \times \mathrm{P} \times \mathrm{Q})($ Hill
\& Hill, 2012). Onde: Z - nível de significância do estudo $=95 \%(1,96) ; \mathrm{P}-$ prevalência do evento = $50 \%$ (utilizou-se 50\%, que maximiza o tamanho da amostra quando não se sabe a prevalência); $\mathrm{Q}$ - complementar da prevalência (1-P); N - população total = 278 (conforme informação do NTI); E - erro amostral de 5\%. Aplicando-se a fórmula, obteve-se um n = 162. Todos os 278 estudantes matriculados foram convidados a participar do estudo, sendo que 147 aceitaram participar e responderam ao instrumento de coleta de dados, ocorrendo assim uma perda amostral de 9,2\%.

\section{Instrumentos}

Foi elaborado um formulário no aplicativo Google Forms contendo: questionário de caracterização, Escala de Depressão, Ansiedade e Estresse - Versão Reduzida (DASS-21), e a Escala de Solidão Universidade de Califórnia (UCLA-BR) e Escala de Apoio Social do Medical Outcomes Study's Social Support Scale (MOS-SSS).

Questionário de Caracterização (elaboração própria). Constituído por questões fechadas dividido em quatro partes: características sociodemográficas, conexão social, saúde e bem-estar, e impacto da pandemia. As questões foram elaboradas a partir de uma ampla revisão de literatura em bases de dados internacionais, considerando aspectos relacionados às relações sociais e a pandemia de covid-19.

As variáveis sociodemográficas utilizadas foram: sexo, orientação sexual, renda, estado civil, cor da pele e religião (categóricas) e idade (numérica). Na parte conexão social as questões buscavam identificar as redes familiares, de amizade e institucionais (referentes ao trabalho, universidade, participação em atividades religiosas e comunitárias) das quais os estudantes faziam parte. As variáveis categóricas utilizadas na parte conexão social foram: com quem residiam, presença de parceiro, quantidade de amigos, contato com redes familiares, de amigos e institucionais.

Na parte "saúde e bem-estar", foram utilizadas as variáveis categóricas: sinto que faço parte da comunidade acadêmica de enfermagem (pertença), ideação suicida, autoestima, timidez, empatia, uso 
de redes sociais. Foram utilizadas também as variáveis categóricas: ansiedade, depressão, estresse e solidão, obtidas por meio de escalas especificas. A ansiedade, a depressão e o estresse foram obtidos por meio da Escala de Depressão, Ansiedade e Estresse - Versão Reduzida (DASS-21); e a solidão, por meio da Escala de Solidão Universidade de Califórnia (UCLA-BR), ambas descritas a seguir.

Escala de Depressão, Ansiedade e Estresse Versão Reduzida (DASS-21). Desenvolvida originalmente por Lovibond e Lovibond (2004), contém três subescalas de sete itens, totalizando 21 itens, que avaliam a depressão, ansiedade e estresse. As respostas são em escala tipo likert de quatro pontos, de o a 3, sendo o - Não se aplicou de maneira alguma e 3 Aplicou-se muito, ou na maioria do tempo (Vignola, 2013; Vignola \& Tucci, 2014).

Escala de Solidão Universidade de Califórnia (UCLA-BR). Instrumento criado por Russell, Peplau e Cutrona (1980) e validado no Brasil, por Barroso e colaboradores (2016), com 20 itens com opções de resposta em escala likert de quatro pontos, partindo de 1 (nunca) a 4 (frequentemente). O escore pode chegar até 60 pontos, sendo classificado assim: de 0 a 22 pontos - solidão mínima; de 23 a 35 pontos - solidão leve; de 36 a 47 pontos - solidão moderada; e de 48 a 60 pontos - solidão intensa.

Na parte "impactos da pandemia" do instrumento de caracterização buscou-se avaliar o impacto da pandemia nas variáveis referente às redes sociais, saúde e bem-estar. Desta parte do instrumento foi utilizada a variável categórica voltar a residir com os pais.

Escala de Apoio Social do Medical Outcomes Study's Social Support Scale (MOS-SSS). Desenvolvida por Sherbourne e Stewart (1991), contém 19 itens com opções de resposta em escala likert de cinco pontos, foi validada para o Brasil por Griep et al. (2005). Zanini e Peixoto (2016) avaliaram a pertinência dos diferentes modelos fatoriais propostos para MOS-SSS em estudos brasileiros, e concluiram melhor qualidade de ajustamento do modelo composto por quatro fatores (apoio emocional/informacional, interação social, apoio material e apoio social afetivo).

A pontuação é específica para cada dimensão. Para o fator emocional/informacional, cuja pontuação varia de 0-32 pontos, é considerado baixo nivel de percepção de apoio social emocional/ informacional escore de 0-12 pontos; médio nivel de 13-28 pontos; e, alto de 29 a 32 pontos. Para o fator interação social, cuja pontuação varia de 0-16 pontos, é considerado baixo nivel de percepção de apoio social relacionado a interação social escore de 0-6 pontos; médio nível de 7-13 pontos; e, escore alto de 14 a 16 pontos. Para o fator material, cuja pontuação varia de 0-16 pontos, é considerado baixo nivel de percepção de apoio social material escore de 0-6 pontos; médio nivel de 7-13 pontos; e, de 14 a 16 pontos. Para o fator afetivo, cuja pontuação varia de 0-12 pontos, é considerado baixo nível de percepção de apoio social relacionado a interação social escore de 0-4 pontos; médio nivel de 5-10 pontos; e, alto de 11 a 12 pontos (Zanini, Peixoto, \& Nakano; 2018).

O resultado da avaliação é obtido através da soma das pontuações dos itens para cada uma das três subescalas, devendo ser obrigatoriamente multiplicado por dois, para o cálculo da pontuação final e aplicação do corte. A classificação para Depressão, Ansiedade e Estresse, respectivamente é: Normal/Leve: 0-9, 0-7 e 0-14; Mínimo: 10-13, 8-9 e 15-18; Moderado: 14-20, 10-14 e 19-25; Grave: 21-27, 15-19 e 26-33; Muito Grave: >28, >20 e >34 (VIGNOLA, 2013). Ressalta-se que esse instrumento foi validado para o Brasil por Vignola e Tucci (2014) e que apresentou adequada validade e confiabilidade em uma amostra de estudantes universitários (Martins et al., 2019).

Assim, para descrever os tipos de redes das quais os estudantes faziam parte utilizou-se as questões presentes na parte conexão social e impactos da pandemia do instrumento de caracterização. E, as subcategorias da escala MOS-SSS foram utilizadas para descrever o apoio social recebido e para realizar os testes de associação com as demais variáveis sociodemográficas, de saúde e bem-estar. 


\section{Procedimentos}

Os dados foram coletados entre 14 de setembro a 27 de novembro do ano de 2020, por meio de formulário eletrônico, elaborado na plataforma Google Forms. O pesquisador principal obteve a lista com e-mails dos estudantes regularmente matriculados por meio da Coordenação do e encaminhou convite para participar da pesquisa bem como o link para responder ao formulário de coleta de dados, individualmente a cada estudante A pesquisa também foi divulgada semanalmente durante o período da coleta por meio dos e-mails das turmas, Facebook, Instagram e site oficial da escola de enfermagem da universidade em que o estudo foi realizado e o link para responder ao formulário eletrônico também foi disponibilizado por esses canais de comunicação. Enquanto não se obteve retorno, o link foi reenviado para o e-mail individual do estudante, até, no máximo, cinco reenvios.

Os estudantes que consentiram em participar, assim que leram e deram anuência, automaticamente assinaram o termo de consentimento livre e esclarecido (TCLE) eletrônico, ao clicar em campo especifico "Li e concordo em participar da pesquisa", requisito para abrir as questões do estudo. Uma via eletrônica do TCLE foi encaminhada para o e-mail dos participantes. Os formulários respondidos eram retornados para a conta do pesquisador no Google Forms.

\section{Análise de dados}

Os dados utilizados foram tabulados em uma planilha do programa Microsoft $®$ Excel (2013), e avaliada quanto à inconsistência dos dados por um segundo pesquisador (a tabela foi conferida para verificação de possiveis erros ou falhas na primeira digitação), e posteriormente foram transferidos para o software Statistical Package for the Social Sciences (SPSS) versão 21.0.

Para as variáveis categóricas foi realizada a descrição da frequência absoluta e frequência relativa. Para as variáveis numéricas foram calculadas as medidas de tendência central (média ou mediana) e medidas de dispersão (desvio padrão - DP).

Foi realizada uma análise estatística inferencial, através de testes estatísticos para verificar a associação entre as variáveis.

O teste quiquadrado foi utilizado para verificar a associação entre as subcategorias da escala MOS-SSS (apoio emocional, interação social, apoio material e apoio afetivo) nos niveis baixo, médio e alto e variáveis categóricas dicotômicas: sinto-me socialmente aceito, ideação suicida, autoestima elevada, timidez, empatia, uso das redes sociais.

O teste de Kolmogorov-Smirnov foi aplicado para verificação da normalidade dos dados numéricos. Como os dados não seguiam uma distribuição normal foi utilizado o teste não paramétrico Mann-Whitney para a variáveis dicotômicas: sexo e religião. E, o teste de Kruskall Walis para as variáveis categóricas com três ou mais categorias: renda, estado civil, orientação sexual, cor da pele.

O Coeficiente de Correlação de Spearman foi utilizado para verificar a correlação entre variáveis numéricas (idade, pontuação final nas subescalas DASS-21 de estresse, ansiedade e depressão e das subescalas MOS-SSS de apoio emocional, interação social, apoio material e apoio afetivo). Em todos os testes foi considerado como estatisticamente significante o valor de $p<0,05$.

O Alpha de Cronbach da escala de solidão da UCLA neste estudo foi $\alpha=0,95$; o da MOS-SSS foi $\alpha=0,97$; e o da DASS-21 foi $\alpha=0,95$. Todas as escalas apresentaram uma boa consistência interna, acima do mínimo recomendado que é $\alpha=0,70$ (Barros et al., 2012).

\section{Resultados}

Como pode ser visualizado na Tabela 1, houve predominância de participantes do sexo feminino (93,2\%), heterossexual (82,3\%), com renda entre um e dois salários-mínimos (42,2\%), autodeclarados brancos (77,6\%), praticantes de alguma religião (63,3\%\%). A média de idade foi de 26,8 anos (DP $\pm 7,8$ ). 
Tabela 1 - Características sociodemográficas dos participantes do estudo. Rio Grande. RS/ Brasil, 2020

\begin{tabular}{|c|c|c|}
\hline $\begin{array}{c}\text { Caracteristicas } \\
\text { Sociodemográficas }\end{array}$ & $\begin{array}{c}n \\
(147)\end{array}$ & $\begin{array}{c}\% \\
(100 \%)\end{array}$ \\
\hline \multicolumn{3}{|l|}{ Sexo } \\
\hline Feminino & 137 & 93,2 \\
\hline Masculino & 10 & 6,8 \\
\hline \multicolumn{3}{|l|}{ Orientação Sexual } \\
\hline Heterossexual & 121 & 82,3 \\
\hline Homossexual & 9 & 6,1 \\
\hline Bissexual & 14 & 9.5 \\
\hline Outros & 3 & 2,1 \\
\hline \multicolumn{3}{|l|}{ Renda } \\
\hline$<1$ Salário & 14 & 9,5 \\
\hline Entre 1 e 2 Salários & 62 & 42,2 \\
\hline 3 Salários & 36 & 24,5 \\
\hline > 3 Salários & 35 & 23,8 \\
\hline \multicolumn{3}{|l|}{ Estado Civil } \\
\hline Solteiro & 100 & 68,0 \\
\hline Casado & 46 & 31,3 \\
\hline Viúvo & 1 & 0,7 \\
\hline \multicolumn{3}{|l|}{ Cor da Pele } \\
\hline Branca & 114 & 77,6 \\
\hline Preta & 15 & 10,2 \\
\hline Parda & 18 & 12,2 \\
\hline \multicolumn{3}{|l|}{ Religião } \\
\hline Não Tem & 54 & 36,7 \\
\hline Pratica alguma religião & 93 & 63.3 \\
\hline
\end{tabular}

Observou-se que 131 participantes (89,1\%) informaram residir com familia nuclear, nove $(6,1 \%)$ residem sozinhos, cinco (3,4\%) com amigos/ colegas, um $(0,7 \%)$ na casa do estudante e um $(0,7 \%)$ em uma comunidade religiosa.

Com relação à existência de um parceiro, 99 $(67,3 \%)$ responderam afirmativamente. Em relação à quantidade de amigos, $60(40,8 \%)$ relataram ter uma boa quantidade de amigos, 70 (47,6\%) poucos amigos e $17(11,6 \%)$ quase nenhum amigo.

Em função da pandemia, 24 (16,3\%) tiveram que voltar a residir com os pais ou familiares. Dentre os 147 estudantes, 103 (70,1\%) ficaram em contato com a rede de familiares e amigos durante a pandemia; 112 (76,2\%) mantiveram contato com os professores da universidade durante a pandemia. Em relação à ocupação, 69 (46,9\%) referiram que apenas estudam, 30 (20,4\%) estudam e trabalham em casa, 29 (19,7\%) estudam e trabalham fora de casa, 19 (12,9\%) estudam e têm algum tipo de bolsa remunerada da universidade.

Em relação à percepção do nível de apoio social recebido por estudantes universitários durante a pandemia, a maioria, 56,5\%, teve médio apoio emocional, 47,6\%, média interação social, $41,5 \%$, alto apoio material, $51,7 \%$, alto apoio afetivo, conforme Tabela 2.

Tabela 2 - Percepção dos participantes do estudo em relação ao nivel de apoio recebido conforme subescalas da escala MOS-SSS. Rio Grande. RS/Brasil, 2020

\begin{tabular}{ccccc}
\hline Nivel & $\begin{array}{c}\text { Apoio Emocional } \\
\mathbf{n}(\%)\end{array}$ & $\begin{array}{c}\text { Interação Social } \\
\mathbf{n}(\%)\end{array}$ & $\begin{array}{c}\text { Apoio Material } \\
\mathbf{n}(\%)\end{array}$ & $\begin{array}{c}\text { Apoio afetivo } \\
\mathbf{n}(\%)\end{array}$ \\
\hline Baixo & $24(16,3 \%)$ & $16(10,9 \%)$ & $27(18,4 \%)$ & $9(6,1 \%)$ \\
Médio & $83(56,5 \%)$ & $70(47,6 \%)$ & $59(40,1 \%)$ & $62(42,2 \%)$ \\
Alto & $40(27,2 \%)$ & $61(41,5 \%)$ & $61(41,5 \%)$ & $76(51,7 \%)$ \\
\hline Total & $147(100 \%)$ & $147(100 \%)$ & $147(100 \%)$ & $147(100 \%)$ \\
\hline
\end{tabular}

Houve correlação negativa com significância estatistica no teste de Spearman somente entre as subescalas da escala MOS-SSS interação social e a idade $(\rho=-177$ e $p<0,032)$. Sendo que quanto maior a idade menor a pontuação na subescala interação social. Não houve diferença estatística significativa $(p<0,05)$ nos testes de comparação das medianas das subescalas da escala MOS-SSS apoio emocional, interação social, apoio material e apoio afetivo para as categorias das variáveis sexo, estado civil e religião. 
Houve diferença estatística significativa no teste de comparação das medianas da subescala da escala MOS-SSS apoio afetivo para as categorias das variáveis orientação sexual $(p=0,008)$ e renda $(p=0,023)$. As medianas para orientação sexual na subescala da escala MOS-SSS apoio afetivo foram: heterossexual=11, homossexual=10, bissexual $=7$, outros $=6$. $E$, as medianas para renda na subescala da escala MOS-SSS apoio afetivo foram: < 1 salário-mínimo=7, entre 1 e 2 salários=10, 3 salários $=12,>3$ salários $=11$.

Houve diferença estatística significativa no teste de comparação das medianas de todas as subescalas da escala MOS-SSS para as categorias da variável cor da pele, conforme Tabela 3.

Tabela 3 - Medianas das diferentes categorias da variável cor da pele nos participantes do estudo conforme subescalas da escala MOS-SSS. Rio Grande. RS/Brasil, 2020

\begin{tabular}{lcccc}
\hline Cor da pele & Apoio emocional & Interação social & Apoio Material & Apoio afetivo \\
\hline Valor de p & 0,034 & 0,028 & 0,039 & 0,006 \\
Branca & 23 & 13 & 13 & 11 \\
Parda & 18,5 & 11 & 9 & 7,5 \\
Preta & 15 & 10 & 10 & 7 \\
\hline
\end{tabular}

De acordo com as subescalas DASS-21, 42,9\% dos estudantes apresentam algum nivel de estresse, 42,3\% algum nível de ansiedade e 54,2\% algum nivel de depressão. Conforme Tabela 4. houve correlação negativa com significância estatística no teste de Spearman entre todas as subescalas da escala MOS-SSS e a subescala da DASS-21 depressão. Quanto menor a pontuação nas subescalas MOS-SSS maior a pontuação na subescala de depressão. Também houve asso- ciação entre as subescalas apoio emocional, interação social e apoio afetivo e a subescala ansiedade. Quanto menor a pontuação nas subescalas apoio emocional, interação social e apoio afetivo maior a pontuação na subescala de ansiedade. E ainda, houve associação entre as subescalas apoio emocional, interação social ea subescala estresse. Quanto menor a pontuação nas subescalas apoio emocional e interação social maior a pontuação na subescala de estresse.

Tabela 4 - Coeficiente de correlação de Spearman e valor de p entre subescalas da DASS-21, Escala Solidão da UCLA e subescalas da escala MOS-SSS nos participantes do estudo. Rio Grande. RS/Brasil, 2020

\begin{tabular}{ccccc}
\hline & $\begin{array}{c}\text { Apoio } \\
\text { emocional }\end{array}$ & Interação social & Apoio Material & Apoio afetivo \\
\hline $\begin{array}{c}\text { Subescala DASS-21 } \\
\text { Depressão }\end{array}$ & $\rho=-389$ & $\rho=-380$ & $\rho=-244$ & $\rho=-318$ \\
Subescala DASS-21 & $\rho<0,001$ & $\rho<0,001$ & $p=0,003$ & $p<0,001$ \\
Ansiedade & $\rho=-327$ & $\rho=-300$ & $\rho=-147$ & $\rho=-228$ \\
Subescala DASS-21 & $p<0,005$ & $\rho<0,001$ & $p=0,075$ & $p=0,005$ \\
Estresse & $\rho=-230$ & $\rho=-265$ & $\rho=-117$ & $\rho=-158$ \\
& $p=0,005$ & $p=0,001$ & $p=0,158$ & $p=0,056$ \\
Escala Solidão UCLA & $\rho=-605$ & $\rho=-587$ & $\rho=-406$ & $\rho=-560$ \\
& $p<0,001$ & $p<0,001$ & $p<0,001$ & $p<0,001$ \\
\hline
\end{tabular}




\section{$8 / 14 \quad$ Psico, Porto Alegre, v. 52, n. 3, p. 1-14, jul.-set. 2021 | e-39894}

Houve correlação negativa com significância estatística no teste de Spearman entre a pontuação final na escala de solidão da UCLA e a pontuação em todas subescalas da MOS-SSS. Sendo que quanto maior a pontuação na subescala UCLA menor a pontuação nas subescalas da MOS-SSS

Houve associação estatística significativa no teste quiquadrado entre as subescalas da escala MOS-SSS interação social e apoio afetivo e a variável sinto que faço parte da comunidade acadêmica da enfermagem, conforme Tabela 5. Houve associação estatística significativa no teste quiquadrado entre as subescalas da escala MOS-SSS e a variável ideação suicida conforme Tabela 6. No entanto, não houve associação estatística significativa no teste quiquadrado entre as variáveis autoestima, empatia, timidez, uso de redes sociais e as subescalas da escala MOS-SSS.

Tabela 5 - Relação entre as subescalas da escala MOS-SSS e sentir-se parte da comunidade acadêmica da enfermagem nos participantes do estudo. Rio Grande. RS/Brasil, 2020

Sinto que faço parte da comunidade acadêmica da enfermagem

\begin{tabular}{lccc} 
Dimensões & \multicolumn{1}{c}{ Sim } & Valor \\
MOS-SSS & $n=89(60,5 \%)$ & $n=58(39,5 \%)$ & de $p$ \\
& \cline { 2 - 4 } & &
\end{tabular}

\section{Apoio Emocional}

$\begin{array}{ccc}\text { Baixo } & 8(9,0 \%) & 16(27,6 \%) \\ \text { Médio } & 47(52,8 \%) & 36(62,1 \%) \\ \text { Alto } & 34(38,2 \%) & 6(10,3 \%)\end{array}$

Interação Social

$\begin{array}{cc}\text { Baixo } & 3(3,4 \%) \\ \text { Médio } & 38(42,7 \%) \\ \text { Alto } & 48(53,9 \%)\end{array}$
$13(22,4 \%)$
$32(55,2 \%)$
$13(22,4 \%)$

Apoio Material

$\begin{array}{ccc}\text { Baixo } & 11(12,4 \%) & 16(27,6 \%) \\ \text { Médio } & 33(37,0 \%) & 26(44,8 \%) \\ \text { Alto } & 45(50,6 \%) & 16(27,6 \%)\end{array}$

Apoio Afetivo

$\begin{array}{ccc}\text { Baixo } & 3(3,4 \%) & 6(10,3 \%) \\ \text { Médio } & 26(29,2 \%) & 36(62,1 \%) \\ \text { Alto } & 60(67,4 \%) & 16(27,6 \%)\end{array}$


Tabela 6 - Relação entre as subescalas da escala MOS-SSS e ideação suicida nos participantes do estudo. Rio Grande. RS/Brasil, 2020

\begin{tabular}{|c|c|c|c|}
\hline \multirow{2}{*}{ Dimensões MOS-SSS } & \multicolumn{2}{|c|}{ Ideação suicida } & \multirow{2}{*}{ Valor de $p$} \\
\hline & $\underset{n=58(39,5 \%)}{\operatorname{Sim}}$ & $\begin{array}{c}\text { Não } \\
n=89(60,5 \%)\end{array}$ & \\
\hline Apoio Emocional & & & $<0,001$ \\
\hline Baixo & $18(31,0 \%)$ & $6(6,7 \%)$ & \\
\hline Médio & $30(51,7 \%)$ & $53(59,6 \%)$ & \\
\hline Alto & $10(17,2 \%)$ & $30(33,7 \%)$ & \\
\hline Interação Social & & & $<0,001$ \\
\hline Baixo & $13(22,4 \%)$ & $3(3,4 \%)$ & \\
\hline Médio & $31(53,4 \%)$ & $39(43,8 \%)$ & \\
\hline Alto & $14(24,1 \%)$ & $47(52,8 \%)$ & \\
\hline Apoio Material & & & 0,009 \\
\hline Baixo & $16(27,6 \%)$ & $11(12,4 \%)$ & \\
\hline Médio & $26(44,8 \%)$ & $33(37,1 \%)$ & \\
\hline Alto & $16(27,6 \%)$ & $45(50,6 \%)$ & \\
\hline Apoio Afetivo & & & $<0,001$ \\
\hline Baixo & $7(12,1 \%)$ & $2(2,2 \%)$ & \\
\hline Médio & $33(56,9 \%)$ & $29(32,6 \%)$ & \\
\hline Alto & $18(31,0 \%)$ & $58(65,2 \%)$ & \\
\hline
\end{tabular}

\section{Discussão}

Observou-se que as redes sociais que os estudantes de enfermagem acessaram durante o periodo de estudo e ao mesmo tempo na pandemia foram: redes familiares, redes de amizade, e redes institucionais, uma vez que, os estudantes permaneceram em contato com seus familiares e amigos, professores da universidade e colegas de trabalho. As redes sociais identificadas neste estudo também foram encontradas em uma tipologia das redes sociais, descrita em um estudo com idosos portugueses, sendo as redes familiares mais frequentes (61,8\%), seguidas das redes de amizade (23.5\%), redes de vizinhança (11,9\%), e por fim, a rede institucional (2,8\%) (Guadalupe \& Vicente, 2020).

Considera-se que as tipologias das redes sociais são baseadas em uma grande diversidade de variáveis de rede (Guadalupe \& Vicente, 2020). Em geral, em estudantes universitários, observa-se que um tamanho maior da rede social é diretamente associado a um maior apoio social e maiores benefícios à saúde (Pokhrel et al, 2016). Evidencia-se ainda que as redes sociais no meio universitário tendem a se apresentar como estruturas globais que representam claramente a estrutura da comunidade (Liu et al., 2017).

A avaliação das redes sociais pode revelar o nivel de integração social de um individuo (Sherbourne \& Stewart, 1991). Isto é particularmente relevante, pois os pais, sobretudo a mãe, e os amigos, são os principais apoiadores sociais dos estudantes universitários (Almeida et al., 2018; Jantara et al., 2020). Além disso, os professores podem exercer esse papel, mesmo que apenas para minoria dos estudantes (Almeida et al., 2018). 
A maioria dos estudantes permaneceu em contato com suas redes sociais durante a pandemia, no entanto, um quantitativo significativo não manteve contato com os amigos/colegas e outro não manteve contato com os professores. Além disso, em decorrência da pandemia, uma parte dos estudantes de enfermagem tiveram que voltar a residir com os pais ou familiares. Assim, mudanças ocorreram na estrutura das redes sociais, indicando uma nova reorganização das mesmas, significando também possiveis implicações no fornecimento e na percepção do apoio social. O fato de os estudantes precisarem voltar a residir com os pais, por exemplo, pode significar, uma insuficiência de recursos materiais, mas também, uma maior percepção de apoio emocional. Ao mesmo tempo, estudantes que deixaram de ter contato com professores e amigos, provavelmente, necessitaram reorganizar sua dinâmica de apoio social.

Estudo de Elmer et al. (2020) investigou as redes sociais e a saúde mental dos alunos antes e no momento da pandemia covid-19, em abril de 2020. Os autores destacaram mudanças em várias dimensões das redes sociais (interação, amizade, apoio social, coestudos) e indicadores de saúde mental (depressão, ansiedade, estresse, solidão) em duas coortes de universitários suíços no momento pandêmico. Em comparações com uma coorte anterior à pandemia, os autores evidenciaram que as redes de interação e de coestudo se tornaram mais esparsas, e mais alunos estudavam sozinhos. Verificou-se ainda que os niveis de estresse, ansiedade, solidão e sintomas depressivos dos alunos pioraram com a pandemia e que preocupações especificas acerca da covid-19, isolamento em redes sociais, falta de interação e suporte emocional e isolamento físico foram associados a trajetórias negativas de saúde mental (Elmer et al., 2020).

Considera-se que a rede social, ao mesmo tempo, pode ou não fornecer apoio social a um sujeito (Griep et al., 2005). As redes sociais podem promover engajamento social e apego dos individuos aos seus amigos, familia e, mais amplamente, sua comunidade (Gorman \& Sivaganesan, 2007).
No entanto, as mesmas podem ser geradoras de estresse, ou até mesmo, causadoras de isolamento social e solidão (Child \& Albert, 2018). Estudo norte-americano comprovou que o estresse na rede social, isto é, aquele gerado por eventos que ocorrem com a familia, amigos ou entes queridos, é mais frequente do que o autoestresse, sendo associado ao sofrimento psicológico individual (Woods-Giscombé et al., 2015).

Portanto, sugere-se que as modificações nas redes sociais decorrentes da pandemia de covid-19, podem impactar tanto positivamente quanto negativamente na saúde dos estudantes, dependendo principalmente de como se darão as relações sociais no período pandêmico, da diversidade das redes sociais mantidas e da capacidade das mesmas fornecerem apoio social. Não obstante, o momento da pandemia, por si só, está relacionado a fatores estressores que requerem gerenciamento dentro das redes sociais, as quais, também demandam reorganização em resposta a este periodo.

Especificamente neste estudo, observou-se redes sociais diversas, tendo em vista que uma parcela da população estudada declarou estudar, trabalhar ou praticar alguma religião. Ressalta-se que uma rede social composta por diferentes tipos de relacionamento promove maior proteção da saúde dos indivíduos (De Jong- Gierveld \& Van Tilburg, 2016). Em contrapartida, com a pandemia de covid-19, muitas atividades foram suspensas, havendo uma restrição no que se refere ao contato com redes sociais já consolidadas, como é o caso evidenciado na pesquisa dos estudantes que deixaram de se relacionar com os professores e entre os pares, podendo significar uma perda importante nas redes sociais.

No que tange à percepção do nível de apoio social recebido pelos estudantes de enfermagem, verificou-se a existência de médio nivel de apoio social nas dimensões apoio emocional/ informacional e interação social, e alto nivel nas dimensões de apoio material e apoio afetivo. Este estudo apresentou uma porcentagem menor na percepção de apoio social em todas as dimensões, em comparação estudo de Souza (2017), que 
identificou que cerca de $69,3 \%$ dos estudantes universitários pesquisados apresentou médio nivel de apoio social. Esse fato pode ter ocorrido devido às particularidades da população de estudo, devido a diferenças regionais e a especificidade de serem apenas acadêmicos de enfermagem. Além disso, também é possivel que o periodo pandêmico tenha contribuido para esse achado.

A satisfação dos estudantes de enfermagem com o suporte social percebido foi avaliada por Almeida et al. (2018), e verificou-se que a maioria deles referiu alto nivel de satisfação com o apoio social recebido. Neste estudo, houve prevalência de uma alta taxa de percepção do apoio social apenas em relação a dimensão apoio material e apoio afetivo, o que pode indicar satisfação nestas dimensões. As dimensões de interação social e apoio emocional/informacional podem ter sido menores percebidas em decorrência de um reflexo do distanciamento social e demais medidas implementadas que restringem o contato social.

Houve associação entre a dimensão da escala MOS-SSS interação social e a idade dos estudantes, sendo que quanto maior a idade, menor a pontuação na subescala interação social. Com relação a isso, pode se observar que o isolamento social e a solidão se associam à idade das pessoas, que quanto maior a idade das pessoas maior o indice destes (De Jong-Gierveld \& Van Tilburg, 2016). Desse modo, pode ser que estudantes com mais idade tenham uma menor percepção do apoio social nessa dimensão por se sentirem mais isolados e mais solitários no ambiente universitário, no qual a faixa etária de estudantes mais novos predomina, o que pode representar dificuldades na interação social. Contudo, estudos adicionais são recomendados para melhor clareza destes resultados.

Não foram verificadas associações entre as subescalas da escala MOS-SSS apoio emocional, interação social, apoio material e apoio afetivo e as categorias das variáveis sexo, estado civil e religião. Em estudo com estudantes universitários da Turquia se identificou não haver diferença significativa quanto ao sexo dos estudantes (Yavuzer et al., 2018). Entretanto, encontrou-se resultado divergente ao esperado no que se refere ao estado civil, uma vez que estar casado ou em um relacionamento está ligado ao fornecimento de apoio social (Diehl et al., 2018), do mesmo modo, com relação à atividade religiosa, que já apareceu associada com o apoio social em estudo anterior (Griep et al., 2005).

As medianas da subescala da escala MOS-SSS apoio afetivo associou-se com as categorias das variáveis orientação sexual. Pessoas com orientação sexual distinta dos heterossexuais sofrem com o estigma social e podem sentir maior solidão (Anderssen et al., 2020). Assim, faz sentido que esses estudantes tenham uma menor percepção do apoio social.

Além disso, as medianas da subescala da escala MOS-SSS apoio afetivo associaram-se com a renda, ao mesmo passo que as medianas de todas as subescalas da escala MOS-SSS se associaram com as categorias da variável cor da pele. Estudo com universitários norte-americanos identificou que estudantes que tinham características diferentes da maioria dos outros estudantes, como raça ou etnia minoritária e baixo nível socioeconômico, apresentam maior risco de isolamento social (Hefner \& Eisenberg, 2009). Dessa forma, o isolamento social pode levar a uma menor percepção de apoio social nestes estudantes.

Houve associação entre todas as dimensões do apoio social e depressão, assim como entre as dimensões apoio emocional, interação social e apoio afetivo e a ansiedade, e entre as dimensões apoio emocional, interação social e ao estresse. Destacou-se, ainda, associação entre a solidão e todas as dimensões do apoio social, bem como entre o apoio social e a ideação suicida. Ao mesmo tempo, destaca-se relação entre o apoio social e a integração acadêmica.

Assim como nesta pesquisa, estudos atestam a relação negativa entre apoio social, depressão, transtornos mentais comuns, estresse, solidão e ideação suicida (Park et al., 2015; Barroso et al., 2019; Graner et al., 2018; Hidalgo-Rasmussen et al., 2019). No entanto, este estudo não identificou associação entre as variáveis autoestima e empatia, associações relatadas pela literatura (Kim, \& Yoo, 2016; Park et al., 2015). 
Em sintese, as redes sociais e o apoio social se relacionam com aspectos protetores à saúde dos estudantes e se refletem em benefícios, seja na saúde ou na formação dos universitários. A ineficácia do apoio social, por sua vez, está associada a condições negativas, como, por exemplo, a presença de transtornos mentais como a ansiedade, o estresse e a depressão, repercutindo na qualidade de vida e no desempenho acadêmico. Evidencia-se assim, que além da existência das redes sociais, elas precisam estabelecer relações que sejam funcionantes, eficazes e saudáveis (Jantara et al., 2020).

Verifica-se, portanto, que as redes sociais dos estudantes pesquisados, são efetivas e funcionais, ao menos em parte da população estudada. Em contraposição, a associação de uma ou mais dimensões do apoio social com demais variáveis pesquisadas, como a depressão, ansiedade, estresse e ideação suicida, pode significar fragilidade nas redes sociais, caracterizada pela baixa percepção do apoio social pelos participantes, uma vez que isto pode ocorrer devido à falta de fornecimento de apoio social. Outrossim é a modificação da dinâmica das redes sociais em decorrência da pandemia, demarcada por algumas perdas estruturais e reorganização das mesmas.

O presente estudo apresenta como limitações sua abordagem transversal, que não permite estabelecer relações de causa e efeitos e a utilização de escalas de sintomas e não de diagnósticos em saúde mental. Novos estudos são recomendados para, além de compreender a dinâmica das redes sociais e apoio social nos estudantes universitários, desvelar com maior profundidade os impactos da pandemia nestes dois tópicos de pesquisa, pois pressupõe-se que as mudanças nas formas de se relacionar e as modificações geradas nas redes sociais podem ter exercido influência. Além disso, é relevante a utilização de abordagens qualitativas, para compreender estes fenômenos de uma maneira mais subjetiva.

\section{Referências}

Almeida, L. Y. de, Carrer, M. O., Souza, J. de, \& Pillon, S. C. (2018). Evaluation of social support and stress in nursing students. Revista da Escola de Enfermagem da USP, 52, e03405-e03405. https://doi.org/10.15.90/ s1980-220x2017045703405

Anderssen, N., Sivertsen, B., Lønning, K. J., \& Malterud, K. (2020). Life satisfaction and mental health among transgender students in Norway. BMC public health, 20(1), 1-11. https://doi.org/10.1186/s12889-020-8228-5

Barros, M. V., Reis, R. S., Hallal, P. C., Florindo, A. A., \& Farias Júnior, J. C. (2012). Análise de dados em saúde. Midiograf, 307.

Barroso, S. M., Andrade, V. S. D., Midgett, A. H., \& Carvalho, R. G. N. D. (2016). Evidências de validade da Escala Brasileira de Solidão UCLA. Jornal Brasileiro de Psiquiatria, 65(1), 68-75. http://dx.doi.org/10.1590/00472085000000105

Barroso, S. M., Oliveira, N. R. D., \& Andrade, V. S. D. (2019). Solidão e Depressão: Relações com Caracteristicas Pessoais e Hábitos de Vida em Universitários. Psicologia: Teoria e Pesquisa, 35, 1-12. https://doi.or$\mathrm{g} / 10.1590 / 0102.3772 \mathrm{e} 35427$

Resolução n 510, de 07 de abril de 2016 (2016). Dispõe sobre as normas aplicáveis a pesquisas em Ciências Humanas e Sociais. Conselho Nacional de Saúde. http:// conselho.saude.gov.br/resolucoes/2016/Res0510.pdf

Cacioppo, J. T. \& Cacioppo, S. (2014). Social relationships and health: The toxic effects of perceived social isolation. Social and personality psychology compass, 8(2), 58-72. https://doi.org/10.1111/spc3.12087

Child, S. T., \& Albert, M. A. (2018). Social networks and health outcomes: importance for racial and socioeconomic disparities in cardiovascular outcomes. Current Cardiovascular Risk Reports, 12(12), 1-30.

De Jong-gierveld, J., \& Van Tilburg, TG. (2016). Social isolation and loneliness. Encyclopedia of mental health, 4(2), 175-178. http://Www.doi.org/10.1016/B978-0-12397045-9.00118-X

Diehl, K., Jansen, C., Ishchanova, K., \& Hilger-Kolb, J. (2018). Loneliness at universities: determinants of emotional and social loneliness among students. International journal of environmental research and public health, 15(9), 1865. https://doi.org/10.3390/ijerph15091865

Elmer, T., Mepham, K., \& Stadtfeld, C. (2020). Students under lockdown: Comparisons of students' social networks and mental health before and during the COVID-19 crisis in Switzerland. Plos one, 15(7), e0236337. https://doi.org/10.1371/journal.pone.0236337

Gorman, B. K., \& Sivaganesan, A. (2007). The role of social support and integration for understanding socioeconomic disparities in self-rated health and hypertension. Social science \& medicine, 65(5), 958-975. https://doi. org/10.1016/j.socscimed.2007.04.017 
Graner, K. M, Moraes, A. B. A, Torres, A. R, Lima, M. C. P, Rolim, G. S, \& Ramos-Cerqueira, A. T. A. (2018). Prevalence and correlates of common mental disorders among dental students in Brazil. PloS one, 13(9), e0204558. https://journals.plos.org/plosone/article?id=10.1371/ journal.pone.0204558

Griep, R. H. (2003). Confiabilidade e validade de instrumentos de medida de rede social e de apoio social utilizados no Estudo Pró-Saúde [Tese de Doutorado não publicadal Escola Nacional de Saúde Pública, Fundação Oswaldo Cruz, Rio de Janeiro, RJ, Brasil.

Griep, R. H., Chor, D., Faerstein, E., Werneck, G. L., \& Lopes, C. S. (2005). Validade de constructo de escala de apoio social do Medical Outcomes Study adaptada para o português no Estudo Pró-Saúde. Cadernos de Saúde Pública, 21, 703-714. https://Www.scielosp.org/ $\mathrm{pdf} / \mathrm{csp} / 2005 . \mathrm{v} 21 \mathrm{n} 3 / 703-714 / \mathrm{pt}$

Guadalupe, S., \& Vicente, H. T. (2020). Types of Personal Social Networks of Older Adults in Portugal. Social Indicators Research, 147(1) 1-22. https://doi.org/10.1007/ s11205-019-02252-3

Hefner, J., \& Eisenberg, D. (2009). Social support and mental health among college students. American Journal of Orthopsychiatry, 79(4), 491-499. https://doi. org/10.1037/a0016918

Hidalgo-Rasmussen, C. A., Chávez-Flores, Y. V., Yanez-Peñúñuri, L. Y., \& Navarro, S. R. M. (2019). Suicide-related behavior and health-related quality of life among first-year university students in a Mexican university. Ciência \& Saúde Coletiva, 24(10), 3763-3772. http://www. doi.org/10.1590/1413-812320182410.26732017

Hill, M. M., \& Hill, A. (2012). Investigação por questionário (2. ed.). Sílabo. http://hdl.handle.net/10400.2/8497

Holmes, E. A., O'Connor, R. C., Perry, V. H., Tracey, I., Wessely, S., Arseneault, L., ... \& Ford, T. (2020). Multidisciplinary research priorities for the COVID-19 pandemic: a call for action for mental health science. The Lancet Psychiatry, 76), 547-560. https://doi.org/10.1016/S22150366(20)30168-1

Holt-Lunstad, J., Robles, T. F, \& Sbarra, D. A. (2017). Advancing social connection as a public health priority in the United States. American Psychologist, 72(6), 517. http://www.doi.org/10.1037/amp0000103

Jantara, R. D., Abreu, D. P. G., Paula, A. C. S. F. de, Silva Ziani, J. da, Jantara, A., \& Silva Roque, T. de. (2020). Social networks and social support in university students: an integrative review. Research, Society and Development, 9(10), e4709108695-e4709108695. https://doi. org/10.33448/rsd-vgi10.8695

Kim, S. J, \& Yoo, I. Y. (2016). Health Promotion Behavior of Chinese International Students in Korea Including Acculturation Factors: A Structural Equation Model. Asian nursing research, 10(1), 25-31. http://Www.doi. org/10.1016/j.anr.2015.10.008

Liu, T., Yang, L., Liu, S., \& Ge, S. (2017). Inferring and analysis of social networks using RFID check-in data in China. PloS one, 12(6), e0178492. https://doi.org/10.1371/ journal.pone.0178492
Lovibond, S. H, \& Lovibond, P.F. (2004). Manual for the Depression Anxiety Stress Scales, (4nd.). Psychology Foundation.

Martins, B. G., Silva, W. R. D., Maroco, J., \& Campos, J. A. D. B. (2019). Escala de Depressão, Ansiedade e Estresse: propriedades psicométricas e prevalência das afetividades. Jornal Brasileiro de Psiquiatria, 68(1), 32-41. http://dx.doi.org/10.1590/0047-208500000022

Oliveira, N. R., \& Barroso, S. M. (2020). Solidão, depressão e suporte social em estudantes de psicologia. TrabaLho (En) Cena, 5(1), 146-162. https://doi.org/10.20873/ 2526-1487V5N1P146

Park, K. H., Kim, D., Kim, S. K., Yi, Y. H., Jeong, J. H., Chae, J., ...Roh, H. R. (2015). The relationships between empathy, stress and social support among medical students. International journal of medical education, 6 , 103-108. 10.5116/ijme.55e6.0d44

Pokhrel, P., Fagan, P., Cassel, K., Trinidad, D. R., Kaholokula, J. K. A., \& Herzog, T. A. (2016). Social network characteristics, social support, and cigarette smoking among Asian/Pacific Islander young adults. American journal of community psychology, 57(3-4), 353-365. https://doi.org/10.1002/ajcp.12063

Russell, D., Peplau, L. A., \& Cutrona, C. E. (1980). The revised UCLA Loneliness Scale: concurrent and discriminant validity evidence. Journal of personality and social psychology, 39(3), 472-480. https://psycnet.apa. org/doi/10.1037/0022-3514.39.3.472

Sherbourne, C. D., \& Stewart, A. L. (1991). The MOS Social Support Survey. Social Science Medicine, 32(6), 705-714. http://www.ncbi.nlm.nih.gov/pubmed/2035047

UNESCO. 2020. COVID-19 educational disruption and responses. Retrieved July 27, 2020, from https:// en.unesco.org/covid19/educationresponse

Vignola, R. C. B. (2013). Escala de depressão, ansiedade e estresse (DASS): adaptação e validação para o português do Brasil IDissertação de Mestrado, Instituto de Saúde e Sociedade, Universidade Federal de São Paulo, Santos, SP, Brasill. http://repositorio.unifesp.br/ handle/11600/48328

Vignola, R. C. B., \& Tucci, A. M. (2014). Adaptation and validation of the depression, anxiety and stress scale (DASS) to Brazilian Portuguese. Journal of affective disorders, 155, 104-109. https://doi.org/10.1016/i.jad.2013.10.031

Woods-Giscombé, C. L., Lobel, M., Zimmer, C., Wiley Cené, C., \& Corbie-Smith, G. (2015). Whose stress is making me sick? Network-stress and emotional distress in African-American women. Issues in mental health nursing, 36(9), 710-717. https://doi.org/10.3109/01612 $\underline{840.2015 .1011759}$

Yavuzer, Y., Albayrak, G., \& Keldal, G. (2018). Relationship between University Students' Perceived Social Support and Depression Levels: Mediating Effects of Problem-Solving Skills. HU Journal of Education, 33(1), 242-255. https://doi.org/10.16986/HUJE.2017027085 
Zanini, D. S., \& Peixoto, E. M. (2016). Social Support Scale (MOS-SSS): analysis of the psychometric properties via item response theory. Paidéia Ribeirão Preto), 26(65), 359-368. http://dx.doi.org/10.1590/198243272665201612

Zanini, D. S., Peixoto, E. M., \& Nakano, T. D. C. (2018). Escala de apoio social (MOS-SSS): Proposta de normatização com referência nos itens. Trends in Psychology, 26(1), 387-399. https://doi.org/10.9788/tp2018.1-15pt

Zanini, D. S., Peixoto, E. M., \& Nakano, T. D. C. (2018). Escala de apoio social (MOS-SSS): Proposta de normatização com referência nos itens. Trends in Psychology, 26(1), 387-399. http://dx.doi.org/10.9788/tp2018.1-15p

\section{Romario Daniel Jantara}

Mestre e doutorando em Enfermagem pela Universidade Federal do Rio Grande (FURG), em Rio Grande, RS, Brasil.

\section{Daiane Porto Gautério Abreu}

Doutora e mestre em Enfermagem pela Universidade Federal do Rio Grande (FURG), em Rio Grande, RS, Brasil; professora da Universidade Federal do Rio Grande (FURG), em Rio Grande, RS, Brasil.

\section{Leni de Lima Santana}

Doutora e mestre em Enfermagem pela Universidade Federal do Paraná (UFPR), em Curitiba, PR, Brasil; professora do Instituto Federal do Paraná (IFPR), em Curitiba, PR, Brasil.

\section{Diéssica Roggia Piexak}

Doutora e mestre em Enfermagem pela Universidade Federal do Rio Grande (FURG), em Rio Grande, RS, Brasil; professora da Universidade Federal do Rio Grande (FURG), em Rio Grande, RS, Brasil.

\section{Stella Minasi de Oliveira}

Doutora e mestre em Enfermagem pela Universidade Federal do Rio Grande (FURG), em Rio Grande, RS, Brasil; professora da Universidade Federal do Rio Grande (FURG), em Rio Grande, RS, Brasil.

\section{Endereço para correspondência}

Romario Daniel Jantara

Universidade Federal do Rio Grande

Rua General Osório, s/n

Centro, 96201-900

Rio Grande, RS, Brasil

Os textos deste artigo foram revisados pela Poá Comunicação e submetidos para validação do(s) autor(es) antes da publicação. 